\title{
Correlation between Findings of Optical Coherence Tomography And Optical Coherence Tomography Angiography in Patients with Diabetic Macular Ischemia
}

\author{
MUHAMMAD A. ABOUHAMID, M.Sc.; EL-SAIED E. EL-DESSOUKY, M.D.; \\ MOHAMED H. EL-BRADEY, M.D. and MONA S. EL-KODDOUSY, M.D.
}

The Department of Ophthalmology, Faculty of Medicine, Tanta University

\begin{abstract}
Background: Diabetic Macular Ischemia (DMI) is a variant of diabetic maculopathy that can be overlooked but has serious implication on management of Diabetic Retinopathy (DR) and Diabetic Macular Edema (DME).

Aim of Study: The aim of the study was to study the findings of OCT (optical coherence tomography) and OCTA (optical coherence tomography angiography) in patients with DMI and correlate them.

Patients and Methods: A cross sectional study that included 30 eyes: 20 eyes with DMI as diagnosed by FFA (fundus fluorescein angiography) and 10 healthy eyes as control. Both groups underwent OCT and OCTA imaging. OCT images were assessed for thickness and structural abnormalities. Macular perfusion was quantified using OCTA images by 2 parameters: FAZ (foveal avascular zone) area and VAD (vessel area density) at 2 levels; SCP (superficial capillary plexus) and DCP (deep capillary plexus).
\end{abstract}

Results: A statistically significant difference as regard values of FAZ area and VAD in both SCP and DCP, using OCTA, was found between patients and control groups.

There was a statistically significant correlation between the retinal thickness of ischemic macular areas and SCP FAZ area, SCP VAD and DCP VAD.

DRIL (disorganization of retinal inner layers) was found in $55 \%$ of patients. A statistically significant correlation was found between the presence of DRIL and both SCP FAZ area and SCP VAD $(r=0.755, p<0.001$ and $r=-0.809, p<0.001$, respectively). Outer retinal layers interruption was found in $30 \%$ of patients. The correlation between the presence of interrupted outer retinal layers and DCP FAZ area and DCP VAD was, however, not statistically significant.

Conclusion: OCTA is able to detect and quantify DMI and it can substitute FFA for that purpose. OCT can detect ischemic areas of the macula as these areas appear to be thinner than normal and to show disorganization of its inner retinal layers.

Correspondence to: Dr. Muhammad A. Abouhamid, The Department of Ophthalmology, Faculty of Medicine, Tanta University
Key Words: Optical coherence tomography angiographyDiabetic macular ischemia.

\section{Introduction}

DIABETIC retinopathy is a major cause of visual loss in developed countries which can be attributed to several factors including diabetic maculopathy, vitreous haemorrhage, retinal detachment and neovascular glaucoma [1]

Diabetic retinopathy represents a microvascular lesion that predominantly affects the small vessels in the form of microvascular occlusion and microvascular leakage. The subsequent retinal hypoxia alters the balance between angiogenic and anti angiogenic growth factors promoting an angiogenic response. It also alters the blood retinal barrier resulting in increased vascular permeability and fluid accumulation with secondary disruption of the retinal micro-architecture [2]. Diabetic maculopathy is a leading cause of visual impairment in diabetic patients. It has been broadly categorized into Diabetic Macular Edema (DME) and Diabetic Macular Ischemia (DMI) [3]

Several studies have tried to correlate patients' visual acuity to the status of macular perfusion and the integrity of macular microstructure. Studies have shown that DMI is associated with low visual acuity in its moderate to severe forms [4]. Several parameters have been linked to visual outcome in DME including retinal thickness in addition to macular microstructural integrity [я. The prescence of macular ischemia may have adverse effects on treatment outcome for DME or limit its benefits whether it is intravitreal pharmacotherapy or photocoagulation. The effect of Intravitreal injection of Anti-VEGF agents for DME in the presence of 
DMI, regarding visual acuity, macular perfusion and disease progression is a matter of controversy among clinical studies and reports [6,7]. Thus, assessing the level of macular ischemia provides a valuable information to the clinician regarding disease severity and progression.

Fundus Fluorescein Angiography (FFA) has been the gold standard for diagnosing and grading DMI. It shows enlargement and irregularities of the Foveal Avascular Zone (FAZ), increased perifoveal intercapillary areas and capillary drop-out. For grading DMI; the ETDRS (early treatment diabetic retinopathy study) used 3 parameters: FAZ size, FAZ outlines and capillary loss in the central subfield [3]. However, this grading system is of limited value in real life practice. FFA is an invasive, dye involving, time consuming procedure with potential hazards. It cannot differentiate changes occurring at the level of Superficial Capillary Plexus (SCP) from those occurring at the level of Deep Capillary Plexus (DCP).

Optical Coherence Tomography (OCT) is a perfect method for assessment of macular structural changes. Some studies tried to assess its potential to be used as an alternative method for assessment of changes in macular perfusion but results were contradictory $[\mathbf{8 , 9 ]}$.

Optical Coherence Tomography Angiography (OCTA) is a novel, rapid, non-invasive, non-dye involving technique that uses the contrast generated between static (tissue) signals and motion (blood flow) signals to provide high resolution, three dimensional mapping of both choroidal and retinal microvasculature (demonstrating SCP and DCP individually). It allows automated quantification of the microvasculature and gives us a further look into the changes occurring in macular ischemia at different levels. Unfortunately, OCTA doesn't lack drawbacks. OCTA can only detect motion signals, it doesn't detect leakage or show staining or pooling in contrast to FFA. OCTA could miss some lesions that show slow motion, such as some microaneurysms and fibrotic Choroidal Neovascularization (CNV). The field of view in current OCTA devices is relatively narrow. Using the current technology, OCTA is more prone to artifacts than traditional angiographies [10]

\section{Material and Methods}

The study was a controlled cross sectional study of 30 eyes (including 20 eyes of 20 patients who were diagnosed of having DMI using FFA and 10 control eyes of healthy subjects matched for age and sex) who attended at the outpatient clinic of the Ophthalmology Department, Tanta University during the period from June 2017 to December 2017. The study excluded patients with hazy media or/and poor fixation interfering with good quality FFA, OCT and OCTA images and patients with a history of previous ocular surgery.

The study was approved by the scientific and ethical committee of the faculty of medicine. An informed consent was obtained from all patients. The study and data collection conformed to all local laws and complied with the principles of the Declaration of Helsinki.

\section{Procedure:}

All patients were submitted to detailed history taking, Best Corrected Visual Acuity Testing (BCVA). (Snellen visual acuities were converted to Decimal notation VA for the purposes of statistical analysis), comprehensive eye examination including anterior and posterior segment examination, OCT and OCTA imaging. Dilatation of the pupils using tropicamide $1 \%$ to facilitate imaging was done for all cases.

FFA was done using intravenous injection of Sodium fluorescein dye $10 \%$ using Heidelberg Retinal Angiogram-2 (Heidelberg Engineering, Germany), for those patients who had already undergone FFA using other angiographic devices within the last 1 month, we didn't perform another angiogram and depended on the printed out FFA images and reports. Using FFA, macular ischemia was defined as enlargement or irregularity of FAZ or the presence of areas of capillary dropout in the macular area. Because of the non-universality of the obtained FFA photographs, proper grading of macular ischemia and quantification of FAZ parameters couldn't be achieved.

The OCT and OCTA imaging was performed using DRI OCT Triton, Swept source OCT (Topcon Corp., Japan).

On OCTA imaging, $6 \times 6 \mathrm{~mm}$ scanning areas centered on the fovea were used. Segmentation of the retina and choroid within the macular area was done into 4 areas: The Superficial Capillary Plexus (SCP) that includes the space form the inner border of Internal Limiting Membrane (ILM) to the inner border of Inner Plexiform Layer (IPL), the Deep Capillary Plexus (DCP) that includes the space form the outer border of IPL to the outer border of Inner Nuclear Layer (INL), the outer retina which is normally avascular and extends from Outer Plexiform Layer (OPL) to RPE and the choriocapillaris which is a 20 -gm thick slab starting 
10 mbelow the RPE-Bruch's Membrane (BM) complex. Using OCTA, macular ischemia was defined as enlargement or irregularity of FAZ or the presence of areas of capillary non flow within the macular area that are not continuous with FAZ.

In order to quantify the OCTA findings within the macula, 2 parameters were used: FAZ area in $\mathrm{tm}^{2}$ and vessel area density $(\%)$.

FAZ area measuring was done by outlining the FAZ in both SCP and DCP images by 2 observers (graders) and the area was then estimated by the device. The graders performed the assessments independently and were masked to the results of each other. Reliability analysis was performed by calculating the Intraclass Correlation Coefficient (ICC) between graders. The average (mean) of the measurements of the 2 graders was used in the subsequent analysis.

Vessel Area Density (VAD) is defined as the percentage of the area occupied by vessels in the segmented area (6 X 6 millimeter squares scanned area centered on the fovea). It was then quantified after converting the obtained images into binary forms using Image $\mathbf{J}$ software and analyzing its particles (Image J 1.48v; National Institutes of Health, Bethesda, Maryland, USA).

To differentiate between macular edema and ischemia, we used criteria used by De Carlo et al., [11] which defined edema in OCTA as cystic spaces with regular borders completely devoid of OCTA signal and ischemia as grayish hue (decreased signal) with irregular borders. The en face images were used also for this purpose.

On OCT imaging, Central Foveal Thickness (CFT) and Subfoveal Choroidal Thickness (SFCT) were measured manually using caliber. After proper localization of ischemic macular areas using OCTA, The retinal thickness in the ischemic area was measured manually using caliber and it was assessed in comparison to a corresponding area in the macula that didn't show ischemia on OCTA, expressed in ratio (ischemic to non ischemic macular thickness ratio), see Fig. (1). Retinal thickness was measured using a caliber extending from the inner aspect of ILM till the inner aspect of RPE.

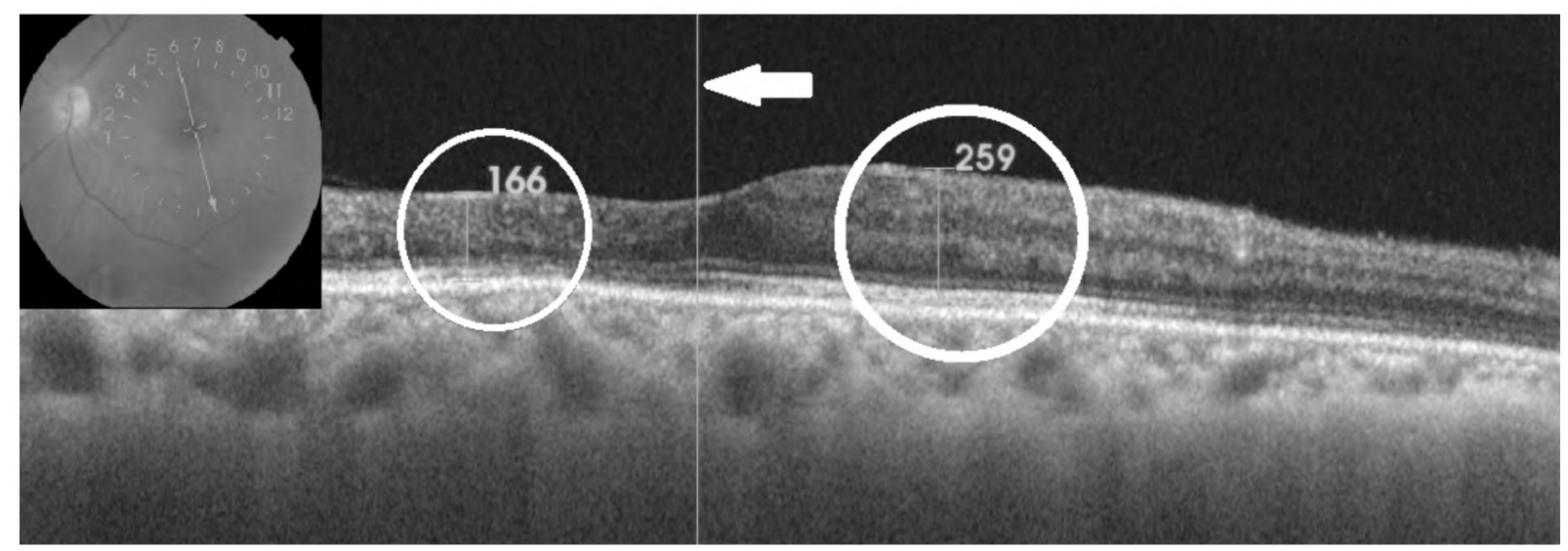

Fig. (1): Ischemic to non ischemic macular thickness ratio estimation, arrow: Line pass through fovea, small circle: Thickness measurement at the ischemic macular area, larger circle: Thickness measurement at a corresponding area of nonischemic macula, ratio $=166 / 259=0.64$.

The ischemic areas seen by OCTA were also evaluated for structural changes using OCT as regard outer retinal and inner retinal changes. Outer retinal changes were defined as any of the following: Interruption or disruption of the ELM and/or Inner Segment-Outer Segment (IS/OS) junction. While inner retinal changes included thinning, or Disorganization of the Retinal Inner Layers (DRIL) which is defined as an area where any boundaries between GCL, IPL, INL, and OPL were poorly identified in the macular scans.

\section{Statistical analysis:}

Analysis of data was performed by using Statistical Package for Social Sciences (SPSS) Version
20 (Chicago, Illinois, USA) [12]. Qualitative data were described using number and percent. The Kolmogorov-Smirnov test was used to verify the normality of distribution. Quantitative data were described using range (minimum and maximum), mean, standard deviation and median.

The used tests included Chi-square test for categorical variables, to compare between different groups, Fisher's exact test for correction for chisquare when more than $20 \%$ of the cells have expected count less than 5, student $t$-test for normally distributed quantitative variables, to compare between two studied groups and Mann Whitney 
test for abnormally distributed quantitative variables, to compare between two studied groups.

Correlations between different variables were done using pearson correlation coefficient for parametric data and spearman correlation coefficient for non parametric data. $p$-value of $\leq 0.05$ was used as a cut off value for significance of results. Reliability analysis was performed by calculating the Intraclass Correlation Coefficient (ICC) between graders to assess inter-grader variation.

\section{Results}

The studied population included 2 groups: The patients group that included 20 eyes with DMI and the the control group that included 10 normal eyes. The patients group included 13 males $(65 \%)$ and 7 females (35\%), with mean age of 54 years. The control group included 6 males $(60 \%)$ and 4 females $(40 \%)$, with mean age of 49 years.

Among the studied group of patients, the mean BCVA for the patients was 0.06 and the median was 0.05 , ranging from 0.03 to 0.10 (decimal). $10 \%$ of the patients had history of intraocular drug injection, $30 \%$ of them had history of previous laser treatment, $20 \%$ of them had history of both treatments (combined treatment), while $40 \%$ of them were naïve to any type of eye intervention. Using fundus biomicroscopy and FFA images, $80 \%$ of the patients had Proliferative Diabetic Retinopathy (PDR), $10 \%$ of them had severe non proliferative diabetic retinopathy and $10 \%$ of them had moderate non proliferative diabetic retinopathy.

\section{OCT data:}

The mean CFT among patients group was 259.6 microns and in the control group was 171 microns $(p<0.001)$. As regard SFCT, its mean value was 249.6 microns in the patients group and 233 microns in the control group ( $p=0.118)$.

The values of macular ischemic/non ischemic retinal thickness ratio ranged between 0.63 to 0.95 . The mean value was 0.80 .

Macular edema could be detected-in any of its forms-in $100 \%$ of cases in the patients group. Disorganization of Retinal Inner Layers (DRIL) was noticed in 55\% of cases in the patient group, while interruption of the outer retinal layers was noticed in $30 \%$ of cases. This interruption involved the continuity of both ELM and IS/OS in all of these cases.
OCTA data:

There was a statistically significant difference as regard FAZ area in both SCP and DCP (for both observers and for average values as well) between the 2 groups. The difference was higher at the level of SCP. Mean SCP FAZ area in patients vs. normal eyes was $719.25 \mathrm{~m} 274.97$ (ranging from $325 \mathrm{~m}^{2} 1300 \mathrm{~m}$ mos. $255.70 \mathrm{~m}-73.31$ (ranging from $184.5 \mathrm{~m} 390 \mathrm{~m}<0.001$. Mean DCP FAZ, area in patients vs. normal eyes was $677,60 \mathrm{~m}-38.35$ (ranging from $410 \mathrm{~m}-1125$ $\mathrm{m}_{2}$ vs. $372.35 \mathrm{~m} 120.90$ (ranging from 142.5 $\mathrm{m}-595 \mathrm{~m} p<0.001$.

Intraclass Correlation Coefficient (ICC) was estimated for the measured FAZ area for both SCP and DCP in both patients and control groups and its results are shown in (Table 2). ICC values were $0.961,0.761,0.973$ and 0.874 for patients' SCP FAZ area, patients' DCP FAZ area, control group's SCP FAZ area and control group's DCP FAZ area, respectively.

As regard VAD, there was a statistically significant difference between its values for both SCP and DCP in the 2 groups. The difference was higher at the level of SCP. Mean SCP VAD in patients vs. normal eyes was $55.83 \% \pm 4.20$ (ranging from $50.9 \%$ to $63 \%$ ) vs. $63.19 \% \pm 1.16$ (ranging from $61.2 \%$ to $65 \%), p<0.001$. Mean DCP VAD in patients vs. normal eyes was $63.95 \% \pm 2.55$ (ranging from $56.7 \%$ to $67.5 \%$ ) vs. $66.51 \% \pm 2.02$ (ranging from $63.3 \%$ to $70.8 \%), p=0.01$.

Using OCTA, findings of macular ischemia were noted in all cases that was diagnosed of having macular ischemia using FFA. In $25 \%$ of patients with PDR: Neovessels (abnormal vessels above the level of ILM) within the scanned area of the macula (6 X 6mm centered on the fovea)using OCTA-were detected.

\section{The correlation between OCT and OCTA data:}

A statistically significant negative correlation was found between BCVA (decimal) and the degree of macular ischemia using the 2 OCTA parameters of macular perfusion (FAZ area and VAD) for both SCP and DCP; (for SCP FAZ area: $r=-0.751, p=$ $<0.001$ ) (for SCP VAD: $r=0.474, p=0.035$ ) (for DCP FAZ area: $r=-0.550, p=0.012$ ) (for DCP VAD: $r=0.686, p=0.001$ ). Also a statistically significant positive correlation was found between BCVA and the retinal thickness of the ischemic area $(r=0.88$, $p<0.001)$.

There was a statistically significant correlation between the retinal thickness of ischemic macular 
areas and SCP FAZ area $(r=-0.760, p<0.001)$, SCP $\operatorname{VAD}(r=0.606, p=0.005)$ and DCP VAD $(r=0.558$, $p=0.011)$ but it was not statistically significant between it and DCP FAZ area ( $r=0.402, p=0.079)$.

Except for a moderate negative correlation between CFT and SCP FAZ area ( $r=-0.482, p=$ 0.032 ), there were no statistically significant correlation between CFT and other OCTA parameters of macular perfusion, nor between SFCT data and any of OCTA data. This can be attributed to the presence of macular edema, which was presentin any of its forms-in $100 \%$ of the studied patients and most probably confounded these results.

A statistically significant correlation was found between the presence of DRIL and both SCP FAZ area and SCP VAD $(r=0.755, p<0.001$ and $r=$ $-0.809, p<0.001$, respectively). The correlation between the presence of interrupted outer retinal layers and DCP FAZ area and DCP VAD was, however, not statistically significant. (Table 2).
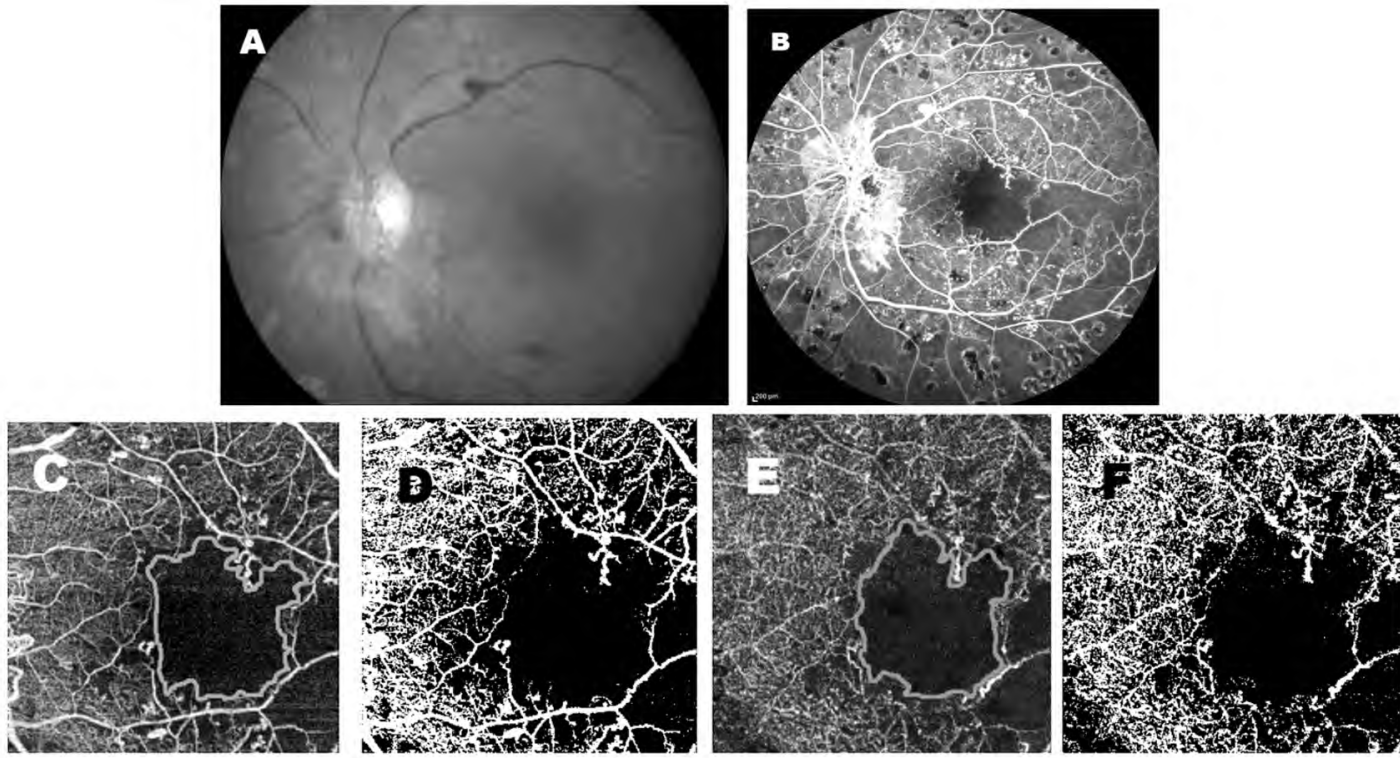

C
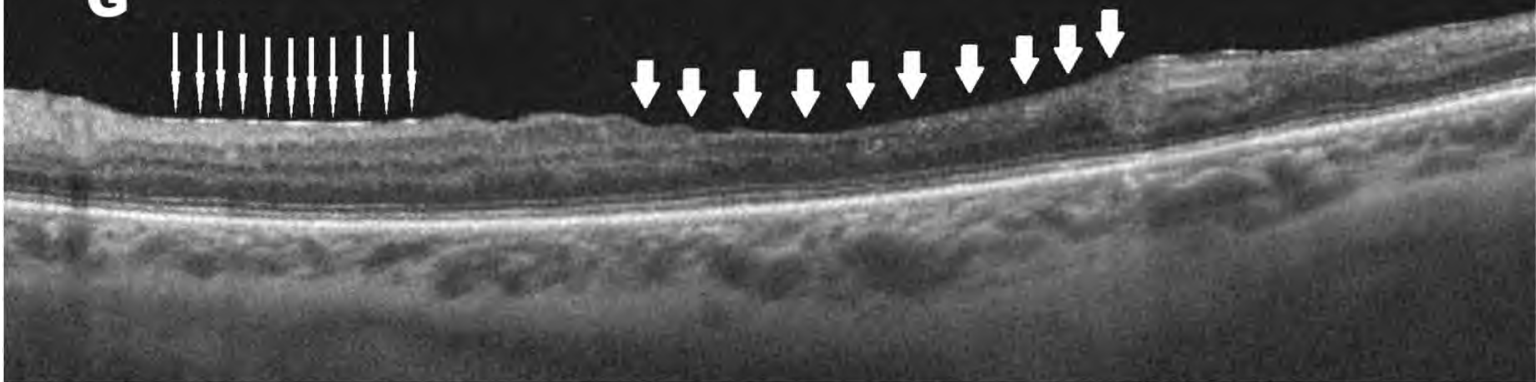

Fig. (2): A 46 years old woman, known to be diabetic for 15 years, with history of previous left eye combined treatment (laser and injection), BCVA (decimal)=0.05. (A): Fundus photo, (B): FFA showing PDR with macular ischemia, (C-F): OCTA images; $\mathrm{C}=$ Outlined SCP FAZ area, D=Binarized image of SCP for VAD estimation, $\mathrm{E}=$ Outlined DCP FAZ area, F=Binarized image of DCP for VAD estimation, (G): SS-OCT; thick arrows: Ischemic area that shows thinning and DRIL, thin arrows: Non ischemic area with preserved organization of inner retinal layers.

Table (1): Correlation between different parameters of OCT and OCTA in patients group.

\begin{tabular}{lcccccccc}
\hline & \multicolumn{2}{c}{$\begin{array}{c}\text { BCVA } \\
\text { (decimal) }\end{array}$} & \multicolumn{2}{c}{$\begin{array}{c}\text { CFT } \\
\text { (microns) }\end{array}$} & \multicolumn{2}{c}{$\begin{array}{c}\text { SFCT } \\
\text { (microns) }\end{array}$} & $\begin{array}{c}\text { Ischemic to non-ischemic } \\
\text { retinal thickness ratio }\end{array}$ \\
\cline { 2 - 10 } & \multicolumn{1}{c}{$p$} & $p$ & $r$ & $p$ & $r$ & $p$ & $r$ & $p$ \\
\hline FAZ area $\left(\mathrm{\mu m}^{2}\right):$ & & & & & & & & \\
$\quad$ SCP & $-0.751^{*}$ & $<0.001$ & $-0.482^{*}$ & 0.032 & 0.054 & 0.821 & $-0.760^{*}$ & $<0.001$ \\
DCP & $-0.550^{*}$ & 0.012 & -0.131 & 0.581 & 0.218 & 0.355 & -0.402 & 0.079 \\
VAD: & & & & & & & & \\
SCP & $0.474^{*}$ & 0.035 & 0.389 & 0.090 & -0.243 & 0.302 & $0.606^{*}$ & 0.005 \\
DCP & $0.686^{*}$ & 0.001 & 0.115 & 0.629 & -0.157 & 0.509 & $0.558^{*}$ & 0.011 \\
\hline
\end{tabular}

$r$ : Pearson coefficient. 
Table (2): Correlation between outer and inner retinal changes with FAZ area and VAD in patients group $(n=20)$.

\begin{tabular}{llccc}
\hline & \multicolumn{2}{c}{$\begin{array}{c}\text { Interrupted } \\
\text { outer retina }\end{array}$} & \multicolumn{2}{c}{ DRIL } \\
\cline { 2 - 5 } & \multicolumn{1}{c}{$p$} & $p$ & $r$ & $p$ \\
\hline FAZ area $\left(\mathbf{m}^{2}\right)$ & & & & \\
SCP & -0.008 & 0.972 & 0.755 & $<0.001^{*}$ \\
DCP & 0.258 & 0.272 & 0.067 & 0.778 \\
VAD: & & & & \\
SCP & -0.057 & 0.810 & -0.809 & $<0.001^{*}$ \\
DCP & -0.337 & 0.146 & -0.268 & 0.253 \\
\hline$r:$ Pearson coefficient. & & & &
\end{tabular}

\section{Discussion}

The current study highlights DMI. A condition that is not fully understood, under diagnosed despite its effect on visual acuity, treatment options and visual prognosis.

In the current study, a statistically significant correlation between BCVA (decimal) and the degree of macular ischemia (evaluated by OCTA using FAZ area and VAD for both SCP and DCP) was found. Also a statistically significant correlation was found between BCVA and the retinal thickness of the ischemic area $(r=0.88, p<0.001)$. This agrees with Samara et al., [9] who found a statistically significant correlation between visual acuity (logMAR) and degree of macular ischemia, evaluated by OCTA.

In the current study, all the patients (100\%) that were agreed to be diagnosed with macular ischemia with FFA showed findings consistent of macular ischemia using OCTA. Comparing both techniques and correlating findings of macular ischemia in FFA and OCTA was not an aim of this study. Some studies tried to compare FFA and OCTA in DMI, including the studies conducted by Garcia et al., [13], Bradley et al., [10] and La et al., [14]. These studies showed a high degree of agreement between FFA and OCTA in evaluating DMI and justified the usage of OCTA in assessing macular perfusion in early diabetic retinopathy.

In the current study, the degree of macular perfusion/ischemia was assessed using two parameters. FAZ area in $\mathbf{m}^{2}$ evaluate FAZ and VAD to quantify macular vascular density. The usage of those 2 parameters was inspired by the study done by Samara et al., [9] who also used FAZ area to evaluate FAZ and used another parameter; Vessel Length Density (VLD) besides VAD to quantify macular vascular density. Other studies included other parameters to evaluate macular perfusion;
Shahlaee et al., [15] used-in addition to FAZ area that showed-according to the same study-the highest inter-observer reliability among the other parameters-FAZ perimeter, maximum vertical and horizontal FAZ diameters to evaluate FAZ. Chu et al., [16] demonstrated a comprehensive quantitative OCTA analysis package for assessment of retinal microvasculature.

In the current study, a statistically significant difference of measurements of average $\mathrm{FAZ}$ area and VAD for both SCP and DCP was found between patients group and the control group (mean $\mathrm{SCP}_{2}$ FAZ area in patients vs ${ }_{2}$ normal eyes $=719.25 \mathrm{rm}^{2}$. \pm 274.97 vs. $255.70 \mathbf{m}^{2}+33.31, p<0.001$ ), (mean $\mathrm{SCP}$ VAD in patients vs. normal eyes $=55.83 \% \pm$ 4.20 vs. $63.19 \% \pm 1.16, p<0.001$ ) (mean DCP FAZ area in patients vs. normal eyes $=677.60$ m 238.35 vs. $372.35 \mathrm{~m}^{2}+120.90, p<0.001$ ), (mean DCP VAD in patients vs. normal eyes $=63.95 \% \pm$ 2.55 vs. $66.51 \% \pm 2.02, p=0.01)$. The study of Samara et al., [9] also showed a statistically significant differences between patients group and a control group but the values showed some difference from our study.

In the current study, FAZ area was greater for both SCP and DCP in patients group than control group. In the patients group, the average $\mathrm{FAZ}$ area of SCP was greater than that of DCP in contrast to the control group where average FAZ area was larger in DCP than SCP. While VAD was greater in the control group than patients group for both SCP and DCP. In both groups, the mean VAD was greater in DCP than SCP. The finding that FAZ area in patients with DMI is greater for SCP than DCP does not agree neither with the above mentioned findings of the study of Samara et al., [9] nor with Freiberg et al., [17] and Minnella et al., [18]. However, the studies conducted by Couturier et al., [19] and Ishibazawa et al., [20] found that capillary non-perfusion areas are larger in the SCP than in the DCP in DR, which agrees with the finding of the current study.

In the current study, FAZ outlining for both SCP and DCP was 2 done by 2 observers and the outlined area in $\mathrm{m}$ generated by the device. The average of the readings of the 2 observers was used for subsequent analysis and intraclass correlation coefficient between the findings of the 2 observers (ICC) was estimated for evaluation of reliability and found to be higher for SCP than DCP and higher for normal eyes than patients eyes (ICC for SCP FAZ area in patients group=0.961), (ICC for SCP FAZ area in control group=0.973), (ICC for DCP FAZ area in patients group=0.761), 
(ICC for DCP FAZ area in control group=0.874). The high variation in DCP FAZ area measurement might be the reason why-when averaging both observers measurements-the average DCP FAZ area in patients group was less than that of SCP FAZ area, despite the opposite was found in control group. ICC of FAZ area in normal eyes in the study of Shahlaee et al., [15] was 0.95 and 0.84 for SCP and DCP respectively. ICC for FAZ area in patients with diabetic retinopathy in the study of Al-Sheikh et al., [21] was 0.997 and 0.992 for SCP and DCP respectively.

In the current study, a statistically significant correlation was found between retinal thickness in the ischemic areas (as shown by OCTA)-expressed in a ratio form between it and the retinal thickness of corresponding non ischemic areas (as seen also by OCTA)-and SCP FAZ area, SCP VAD and DCP VAD but not statistically significant with DCP FAZ area. This finding agrees with the study conducted by Sim et al., [22] that reported that in patients with type $2 \mathrm{DM}$, DMI was associated with macular thinning at different levels. But it also doesn't agree with the study of Reznicek et al., [23] who concluded that total retinal thickness was significantly increased in ischemic compared to non ischemic areas.

In the current study, Disorganization of the Retinal Inner Layers (DRIL) was reported in 55\% of the studied group of patients. The term (DRIL) was first described by Sun et al., [24] as an OCT biomarker and important predictor of vision in eyes with DME. There was a statistically significant correlation between the presence of DRIL and both SCP FAZ area and SCP VAD $(r=0.755, p<0.001$ and $r=-0.809, p<0.001$, respectively). This agrees with the study conducted by Nicholson et al., [25] that reported that DRIL was significantly associated with capillary non perfusion on FFA. Onishi et al., [26] found strong correlation between retinal ischemia at multiple levels and DRIL.

However, in the current study, the correlation between the presence of interruption of outer retinal layers (at level of both ELM and photoreceptors) and DCP parameters (FAZ area and VAD) was non-significant and couldn't be established. In contrast to our study, Lee et al., [27] found a significant correlation between outer retinal abnormalities, especially photoreceptor disruption and the presence of DMI. Scarinci et al., [28] observed that photoreceptor disruption on OCT in eyes with DMI corresponds to areas of capillary non-flow at the level of DCP using OCTA. Byeon et al., [29] found evidence of foveal ganglion cell layer dam- age in DMI. The inability to establish a correlation between outer retinal layer abnormalities and OCTA parameters of macular perfusion, in the current study, could be due to the small number of the patients.

We acknowledge the limitations of the current study. The small number of cases is a major one. Being a cross sectional study and not a prospective one is another. Patients' medical records were not available and data obtained from patients depended mainly on history taking. The results were obtained during a single appointment, and there was no follow-up, we couldn't study the effect of the different treatments that the patients were exposed to on DMI. Enrollment of patients was based on FFA findings which were not conducted by the same device and thus couldn't be quantified or compared and limited the study group to the cases of late DR that appeared with agreement in FFA. The presence of co-exiting DME in all of our cases was a confounder regarding OCT and OCTA interpretation that can't be neglected. Also the patients included in this study underwent different types of previous eye treatments, which might also act as confounding factors.

\section{Conclusion and Recommendations:}

A statistically significant difference was found between patients and control group as regard OCTA parameters of macular ischemia, thus OCTA has the potential to be used alone to detect and quantify DMI.

The statistically significant correlation between the presence of the finding of Disorganization of Retinal Inner Layers (DRIL) by OCT and DMI encourages to recommend that OCT can be used to detect the presence of DMI in cases when FFA cannot be done and OCTA is unavailable.

\section{Financial support and sponsorship:}

Nil.

\section{Conflicts of interest:}

There are no conflicts of interest.

\section{References}

1- FONG D.S., FERRIS F.L., DAVIS M.D. and CHEW E.Y. Causes of severe visual loss in the early treatment diabetic retinopathy study: ETDRS report no. 24. American Journal of Ophthalmology, 127 (2): 137-41, 1999.

2- STEWART M.W.: Pathophysiology of diabetic retinopathy. Diabetic Retinopathy: Springer, p. 1-30, 2010.

3- Group ETDRSR. Classification of diabetic retinopathy from fluorescein angiograms: ETDRS report number 11. Ophthalmology, 98 (5): 807-22, 1991. 
4- SIM D.A., KEANE P.A., ZARRANZ-VENTURA J., FUNG S., POWNER M.B., PLATTEAU E., et al.: The effects of macular ischemia on visual acuity in diabetic retinopathy. Investigative ophthalmology \& visual science, 54 (3): 2353-60, 2013.

5- FURSOVA A., CHUBAR N., TARASOV M., SAIFULLINA I. and PUSTOVAYA G.: Clinical associations between photoreceptor status and visual outcomes in diabetic macular edema. Vestn Oftalmol., 133 (1): 11-8, 2017.

6- CHUNG S.S., HO E.C., LAM K.S. and CHUNG S.K.: Contribution of polyol pathway to diabetes-induced oxidative stress. Journal of the American Society of Nephrology, 14 (suppl 3): S233-S6, 2003.

7- CHANDRA S., SHETH J., ANANTHARAMAN G. and GOPALAKRISHNAN M.: Ranibizumab-induced retinal reperfusion and regression of neovascularization in diabetic retinopathy: An angiographic illustration. American Journal of Ophthalmology Case Reports, 2018.

8- DMUCHOWSKA D.A., KRASNICKI P. and MARIAK Z.: Can optical coherence tomography replace fluorescein angiography in detection of ischemic diabetic maculopathy? Graefe's Archive for Clinical and Experimental Ophthalmology, 252 (5): 731-8, 2014.

9- SAMARA W.A., SHAHLAEE A., ADAM M.K., KHAN M.A., CHIANG A., MAGUIRE J.I., et al.: Quantification of diabetic macular ischemia using optical coherence tomography angiography and its relationship with visual acuity. Ophthalmology, 124 (2): 235-44, 2017.

10- BRADLEY P.D., SIM D.A., KEANE P.A., CARDOSO J., AGRAWAL R., TUFAIL A., et al.: The evaluation of diabetic macular ischemia using optical coherence tomography angiography. Investigative ophthalmology \& visual science, 57 (2): 626-31, 2016.

11- De CARLO T.E., CHIN A.T., JOSEPH T., BAUMAL C.R., WITKIN A.J., DUKER J.S., et al.: Distinguishing diabetic macular edema from capillary nonperfusion using optical coherence tomography angiography. Ophthalmic Surgery, Lasers and Imaging Retina, 47 (2): 108-14, 2016.

12- KIRKPATRICK L.A. and FEENEY B.C.: A simple guide to IBM SPSS: For Version 20.0: Nelson Education, 2012.

13- GARCIA JMBDB, LIMA T.T., LOUZADA R.N., RASSI A.T., ISAAC D.L.C. and AVILA M.: Diabetic macular ischemia diagnosis: Comparison between optical coherence tomography angiography and fluorescein angiography. Journal of Ophthalmology, 2016, 2016.

14- LA A.M., KURT R.A., MEJOR S., EGAN C.A., TUFAIL A., KEANE P.A., et al.: Comparing Fundus Fluorescein Angiography And Swept- source Optical Coherence Tomography Angiography In The Evaluation Of Diabetic Macular Perfusion. Retina (Philadelphia, Pa), 2018.

15- SHAHLAEE A., PEFKIANAKI M., HSU J. and HO A.C.: Measurement of foveal avascular zone dimensions and its reliability in healthy eyes using optical coherence tomography angiography. American Journal of Ophthalmology, 161: 50-5. e1, 2016.

16- CHU Z., LIN J., GAO C., XIN C., ZHANG Q., CHEN C.L., et al.: Quantitative assessment of the retinal microvasculature using optical coherence tomography angiography. Journal of Biomedical Optics, 21 (6): 066008 , 2016.

17- FREIBERG F.J., PFAU M., WONS J., WIRTH M.A.,
BECKER M.D. and MICHELS S.: Optical coherence tomography angiography of the foveal avascular zone in diabetic retinopathy. Graefe's Archive for Clinical and Experimental Ophthalmology, 254 (6): 1051-8, 2016.

18- MINNELLA A.M., SAVASTANO M.C., FEDERICI M., FALSINI B. and CAPOROSSI A.: Superficial and deep vascular structure of the retina in diabetic macular ischaemia: OCT angiography. Acta Ophthalmol. doi., 10, 2016.

19- COUTURIER A., MANÉ V., BONNIN S., ERGINAY A., MASSIN P., GAUDRIC A., et al.: Capillary plexus anomalies in diabetic retinopathy on optical coherence tomography angiography. Retina, 35 (11): 2384-91, 2015.

20- ISHIBAZAWA A., NAGAOKA T., TAKAHASHI A., OMAE T., TANI T., SOGAWA K., et al.: Optical coherence tomography angiography in diabetic retinopathy: A prospective pilot study. American Journal of Ophthalmology, 160 (1): 35-44. e1, 2015.

21- AL-SHEIKH M., AKIL H., PFAU M. and SADDA S.R.: Swept-source OCT angiography imaging of the foveal avascular zone and macular capillary network density in diabetic retinopathy. Investigative ophthalmology \& visual science, 57 (8): 3907-13, 2016.

22- SIM D.A., KEANE P.A., FUNG S., KARAMPELAS M., SADDA S.R., FRUTTIGER M., et al.: Quantitative analysis of diabetic macular ischemia using optical coherence tomography. Investigative ophthalmology \& visual science, 55 (1): 417-23, 2014.

23- REZNICEK L., KERNT M., HARITOGLOU C., KAMPIK A., ULBIG M. and NEUBAUER A.S.: In vivo characterization of ischemic retina in diabetic retinopathy. Clinical Ophthalmology (Auckland, NZ), 5: 31, 2011.

24- SUN J.K., LIN M.M., LAMMER J., PRAGER S., SARANGI R., SILVA P.S., et al.: Disorganization of the retinal inner layers as a predictor of visual acuity in eyes with center-involved diabetic macular edema. JAMA ophthalmology, 132 (11): 1309-16, 2014.

25- NICHOLSON L., RAMU J., TRIANTAFYLLOPOLOU I., PATRAO N.V., COMYN O., HYKIN P.G., et al.: The sensitivity and specificity of disorganisation of the retinal inner layers on SD-OCT in detecting capillary nonperfusion in diabetic retinopathy. Investigative Ophthalmology \& Visual Science, 56 (7): 5940, 2015.

26- ONISHI A.C., ASHRAF M., SOETIKNO B.T. and FAWZI A.A.: Multilevel Ischemia In Disorganization of The Retinal Inner Layers On Projection-resolved Optical Coherence Tomography Angiography. Retina (Philadelphia, Pa), 2018.

27- LEE D.H., KIM J.T., JUNG D.W., JOE S.G. and YOON Y.H.: The relationship between foveal ischemia and spectral-domain optical coherence tomography findings in ischemic diabetic macular edema. Investigative ophthalmology \& visual science, 54 (2): 1080-5, 2013.

28- SCARINCI F., NESPER P.L. and FAWZI A.A.: Deep retinal capillary nonperfusion is associated with photoreceptor disruption in diabetic macular ischemia. American Journal of Ophthalmology, 168: 129-38, 2016.

29- BYEON S.H., CHU Y.K., LEE H., LEE S.Y. and KWON O.W.: Foveal ganglion cell layer damage in ischemic diabetic maculopathy: Correlation of optical coherence tomographic and anatomic changes. Ophthalmology, 116 (10): 1949-59. e8, 2009. 


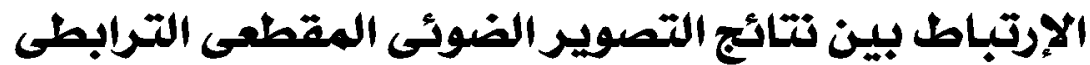

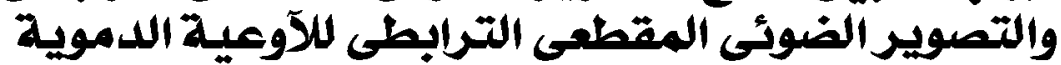

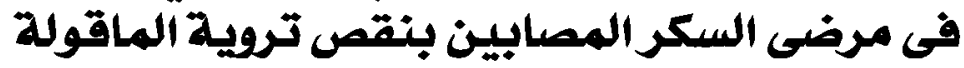

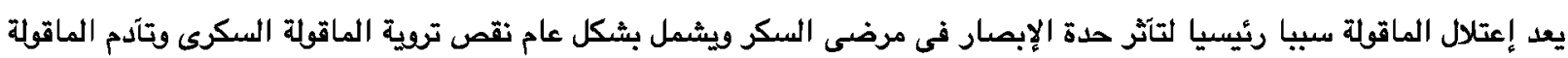

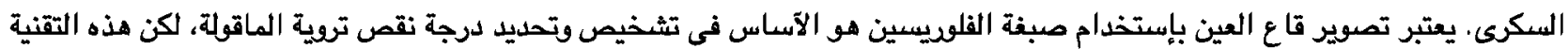

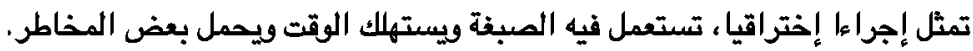

يتيح التصوير الضوئى المقطعى الترابطى المصول على صود عالية الجودة تمثل مقاطع عرضية في الشبكية، ما يجعله وسيلة مثالية

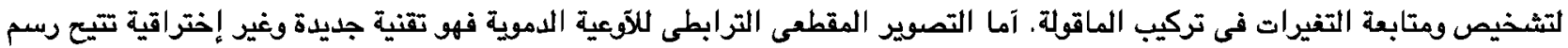

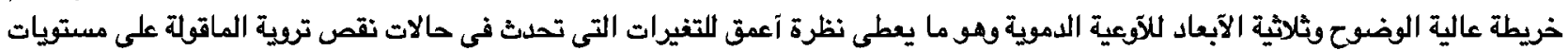

مختلة.

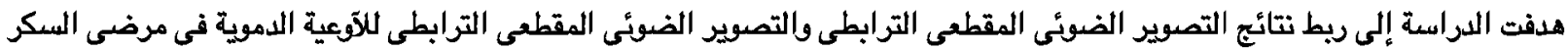

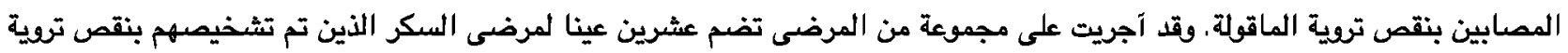

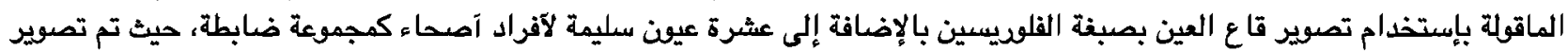
الماقولة فى كلا المجموعتين بإستخدام جهاز التصوير الضوئى المقطعى الترابطى والتصوير الضوئي المقطعى الترابطى لآوعيتها الدموية.

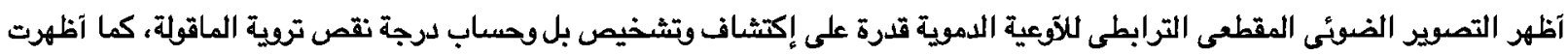

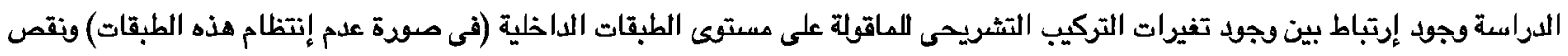

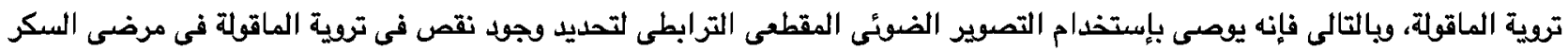

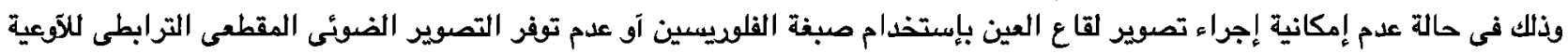

